\title{
Performance Analysis of Maximum-Likelihood Multiuser Detection in Space-Time Coded CDMA with Imperfect Channel Estimation
}

\author{
G. V. V. Sharma and A. Chockalingam \\ Department of ECE, Indian Institute of Science, Bangalore 560012, INDIA
}

\begin{abstract}
In this paper, we analyze the performance of maximumlikelihood (ML) multiuser detection in space-time coded CDMA systems with imperfect channel estimation. A $k$-user synchronous CDMA system which employs orthogonal space-time block codes with $M$ transmit antennas and $N$ receive antennas is considered. A least-squares estimate of the channel matrix is obtained by sending a sequence of pilot bits from each user. The channel matrix is perturbed by an error matrix which depends on the thermal noise as well as the correlation between the signature waveforms of different users. Using the characteristic function of the decision variable, we derive an exact expression, in closed-form, for the pairwise error probability (PEP) of the joint data vector of bits from different users. Using this exact PEP expression, we obtain an upper bound on the average bit error rate (BER). The analytical BER bounds are compared with the BER obtained through simulations. The BER bounds are shown to be increasingly tight for large SNR values. It is shown that the degradation in BER performance due to imperfect channel estimation can be compensated by using more number of transmit/receive antennas.
\end{abstract}

Keywords - ML multiuser detection, space-time codes, CDMA, imperfect channel estimation.

\section{INTRODUCTION}

Space-time coded transmission using multiple transmit antennas can offer the benefits of transmit diversity and high data rate transmission on fading channels [1]. Space-time coding applied to code division multiple access (CDMA) systems has been of interest [2]. Multiuser detection schemes, which can significantly enhance the receiver performance and increase the capacity of CDMA systems, have been extensively studied in the literature, mainly for single transmit antenna systems [3]. Multiuser detection schemes and their performances in space-time coded CDMA systems with multiple transmit antennas has been a topic of recent investigations $[4],[5],[6],[7]$. The performance of the systems considered in [4]-[6] were evaluated mainly through simulations. In [7], Uysal and Georghiades derived an exact analytical expression for the pairwise error probability (PEP) and obtained approximate bit error probability for a space-time coded CDMA system. However, the detector considered in [7] is not a multiuser detector. In [8], Taricco and Biglieri obtained an expression for the PEP of space-time codes in a single user system, assuming perfect channel estimation at the receiver. Using this PEP, they obtained bounds on the probability of error for maximum-likelihood (ML) detection. In [9], Garg et al extended the work in [8] by incorporating imperfect channel

This work was supported in part by the Swarnajayanti Fellowship from the Department of Science and Technology, Government of India, New Delhi under scheme Ref: No. 6/3/2002-S.F estimation in the system model, again for the single user system.

For a multiuser system, bounds on the bit error probability of the maximum-likelihood (ML) multiuser detector have been derived in [3] (Ch. 4.3) for a 1-Tx/1-Rx antenna system. In [10], we cosidered a space-time coded CDMA system with multiple transmit and multiple receive antennas where we derived bounds' on the bit error probability of the ML multiuser detector. The analysis in [10] assumed perfect knowledge of the channel coefficients at the receiver. Our new contribution in this paper is to analyze the performance when the channel estimates at the receiver are imperfect. A least-squares estimate of the channel matrix is obtained by sending a sequence of pilot bits from each user. The channel matrix is perturbed by an error matrix which depends on the thermal noise as well as the correlation between the signature waveforms of different users.

Using a discrete-time vector model of the received signal in a space-time coded CDMA system with $M$ transmit and $N$ receive antennas as in [11], and the characteristic function of the decision variable, we derive an exact expression, in closed-form, for the pairwise error probability (PEP) of the joint data vector of bits from different users. Using this exact PEP expression, we then obtain an upper bound on the average bit error rate (BER). We compare the analytical BER bounds with the BER obtained through simulations, and show that the BER bounds are increasingly tight for large SNR values. It is shown that the degradation in BER performance due to imperfect channel estimation can be compensated by using more number of transmit/receive antennas.

The rest of the paper is organized as follows. In Section II, we present the system model. In Section III, we present the performance analysis. Section IV presents the results and discussions. Conclusions are given in Section V.

\section{SYSTEM MODEL}

Consider a $K$-user synchronous CDMA system with $M$ transmit antennas per user. Users transmit data blocks with $Q$ bits per data block. Let $b_{i q}, i \in\{1,2, \ldots, K\}, q \in\{1,2, \ldots, Q\}$, be the $q^{\text {th }}$ bit of the $i^{\text {th }}$ user, transmitted in a time interval of length $T$. The bits in a data block are mapped on to the $M$ transmit antennas using orthogonal space-time block codes (STBC) [12]. We assume that the channel fading is quasistatic over one data block. Let $A_{i p}$ be the transmit amplitude on the $p^{\text {th }}$ transmit antenna of the $i^{\text {th }}$ user, $h_{i p}$ the complex channel gain from the $p^{\text {th }}$ transmit antenna of the $i^{\text {th }}$ user, and $s_{i}$ represent the unit energy signature waveform of the $i^{t h}$ user. The demodulator on each receive antenna uses a bank of 
$K$ matched filters, each matched to a different user's signature waveform. Assuming that the signature waveforms of different users are synchronous, the received signal at the output of the $j^{\text {th }}$ matched filter for the $q^{\text {th }}$ data bit is denoted as $y_{j q}$, where $j=1,2, \ldots, K$, and $q \in\{1,2, \ldots, Q\}$. We define matrix $\mathbf{R}$ as

$$
\mathbf{R}=\left[\begin{array}{cccc}
1 & \rho_{12} & \ldots & \rho_{1 K} \\
\rho_{12} & 1 & \ldots & \rho_{2 K} \\
\vdots & \vdots & \vdots & \vdots \\
\rho_{1 K} & \rho_{2 K} & \ldots & 1
\end{array}\right]
$$

where $\rho_{j k}=\left\langle s_{i}, s_{j}\right\rangle$ is the inner product of the signature sequences of the $i^{\text {th }}$ and $j^{\text {th }}$ users over the time interval $T$. Defining

$\mathbf{H}_{q}=\operatorname{diag}\left[A_{1 q} h_{1 q}, \ldots, A_{K^{i} q} h_{K q}\right], \mathbf{y}_{q}=\left[y_{1 q}, \ldots, y_{K q}\right]^{T}$, $\mathbf{y}=\left[\mathbf{y}_{1}^{T}, \mathbf{y}_{2}^{T}, \ldots, \mathbf{y}_{Q}^{T}\right]^{T}, \mathbf{b}_{q}=\left[b_{1 q}, \ldots, b_{k q}\right]^{T}$ and

$\mathbf{b}=\left[\mathbf{b}_{1}^{T}, \ldots, \mathbf{b}_{Q}^{T}\right]^{T}$, the received signal vector $\mathbf{y}$ can be written in the form [11]

$$
\mathbf{y}=\mathbf{C H b}+\boldsymbol{\eta},
$$

where the correlation matrix, $\mathbf{C}$, is given by

$$
\mathbf{C}=\left[\begin{array}{ccccc}
\mathbf{R} & \mathbf{0} & \mathbf{0} & \mathbf{0} & \ldots \\
\mathbf{0} & \mathbf{R} & \mathbf{0} & \mathbf{0} & \ldots \\
\mathbf{0} & \mathbf{0} & \mathbf{R} & \mathbf{0} & \ldots \\
\vdots & \vdots & \vdots & \vdots & \vdots \\
\mathbf{0} & \ldots & \mathbf{0} & \mathbf{0} & \mathbf{R}
\end{array}\right],
$$

and $E\left[\eta \eta^{\dagger}\right]=2 \sigma^{2} \mathbf{C}$, where $\eta^{\dagger}$ denotes the Hermitian of the complex vector $\eta$. The channel matrix $\mathbf{H}$ for $M=Q=8$ is given by

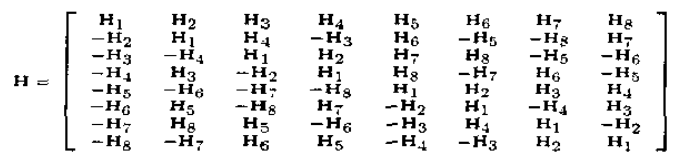

The vector model in (2) can be valid for other values of $M$, provided the matrices, $\mathbf{H}$ (of order $Q K \times Q K$ ) are defined appropriately. For values of $M$ and $Q$ other than $8(M, Q<8)$, $\mathbf{H}$ is given by the upper leftmost submatrix of order $Q K \times Q K$ in (4). For the case of $M \notin\{1,2,4,8\}, M<Q$. Therefore, only the elements $\mathbf{H}_{q}, q=1,2, \ldots, M$, are non-zero, i.e., $\mathbf{H}_{q}=\mathbf{0}$ for $M<q \leq Q$. The entries of the channel matrix $\mathbf{H}$ are assumed to be i.i.d, zero-mean complex circular Gaussian random variables (Rayleigh fading). Assuming the correlation matrix $\mathbf{C}$ to be positive definite, we do the Cholesky decomposition of $\mathbf{C} \quad \mathbf{C}=\mathbf{F}^{\mathcal{T}} \mathbf{F}$.

$$
\widehat{\mathbf{y}}=\left(\mathbf{F}^{T}\right)^{-1} \mathbf{y}=\mathbf{F H b}+\mathbf{n},
$$

where $E[\mathbf{n}]=0_{Q K \times 1}, E\left[\mathbf{n n}^{\dagger}\right]=2 \sigma^{2} \mathbf{I}_{Q K}$, where $(.)^{\dagger}$ represents the Hermitian operation and $I$ is the identity matrix.

\section{A. Channel Estimation}

Each user is assumed to transmit a sequence of $Q$ pilot bits $L_{p}$ for the purpose of channel estimation at the receiver. From (6), the received vector due to the $k^{\text {th }}$ set of $Q$ pilot bits per user is obtained as

$$
\widehat{\mathbf{y}}_{k}=\mathbf{F H} \mathbf{b}_{k}+\mathbf{n}_{k}, 1 \leq k \leq L_{p},
$$

Let the matrix $\mathbf{B}_{p}$ of dimension $Q K \times L_{p}$ denote the sequence of composite pilot vectors $\mathbf{b}_{1}, \mathbf{b}_{2}, \cdots, \mathbf{b}_{L_{p}} . \mathbf{B}_{p}$ is given by

$$
\mathbf{B}_{p}=\left[\begin{array}{llll}
\mathbf{b}_{1} & \mathbf{b}_{2} & \cdots & \mathbf{b}_{L_{p}}
\end{array}\right],
$$

where $\mathbf{n}_{1}, \mathbf{n}_{2}, \cdots, \mathbf{n}_{L_{p}}$ are complex Gaussian random vectors such that

$$
E\left[\mathbf{n}_{p}\right]=\mathbf{0}_{Q K \times 1}, E\left[\mathbf{n}_{p} \mathbf{n}_{p}^{\dagger}\right]=2 \sigma^{2} I_{Q K} .
$$

The received pilot matrix $\widehat{\mathbf{Y}}_{p}$ can then be written as

$$
\widehat{\mathbf{Y}}_{p}=\mathbf{F H B}_{p}+\mathbf{N}_{p} \text { : }
$$

where $\mathbf{N}_{p}=\left[\begin{array}{llll}\mathbf{n}_{1} & \mathbf{n}_{2} & \cdots & \mathbf{n}_{L_{p}}\end{array}\right]$.

The least-squares estimate of the channel matrix $\mathbf{H}$ can be obtained as

$$
\widehat{\mathbf{H}}=\mathbf{F}^{-1} \widehat{\mathbf{Y}}_{p} \mathbf{B}_{p}^{T}\left(\mathbf{B}_{p} \mathbf{B}_{p}^{T}\right)^{-1} .
$$

For the above equation to hold, the matrix $\left(\mathbf{B}_{p} \mathbf{B}_{p}^{T}\right)$ has to be invertible, i.e., $L_{p} \geq Q K$. From (10) and (11),

$$
\widehat{\mathbf{H}}=\mathbf{H}+\mathbf{F}^{-1} \mathbf{N}_{p} \mathbf{B}_{p}^{T}\left(\mathbf{B}_{p} \mathbf{B}_{p}^{T}\right)^{-1} .
$$

\section{B. ML Criterion}

Using the vector representation of the multiuser received signal in (6), the maximum-likelihood (ML) multiuser detection criterion can be written as follows. From (11), we obtain the estimates of the channel gains at the receiver. The ML estimate of the transmitted bit vector, $\mathbf{b}$, (comprising the bits from all users) is then given by

$$
\tilde{\mathbf{b}}=\arg \left\{\min _{\mathbf{x}} \sum_{j=1}^{N}\left\|\widehat{\mathbf{y}}^{(j)}-\mathbf{F} \widehat{\mathbf{H}}^{(j)} \mathbf{x}\right\|^{2}\right\},
$$

where the superscript $(j)$ in $\mathbf{y}$ and $\widehat{\mathbf{H}}$ denote the receive antenna index, and the $\min _{\mathbf{x}}$ is over all possible bit vectors of length $Q K$. Substituting (6) in (13)

$\tilde{\mathbf{b}}=\arg \left\{\min _{\mathbf{x}} \sum_{j=1}^{N}\left\|\mathbf{F} \mathbf{H}^{(j)}(\mathbf{b}-\mathbf{x})+\mathbf{n}^{(j)}-\mathbf{N}_{p}^{(j)} \mathbf{B}_{p}^{T}\left(\mathbf{B}_{p} \mathbf{B}_{p}^{T}\right)^{-1} \mathbf{x}\right\|^{2}\right\}$.

\section{Performance Analysis}

In this section, we analyze the bit error performance of the ML multiuser detection scheme in (14). We first derive an expression for the pairwise error probability (PEP), $P(\mathbf{b} \rightarrow$ $\tilde{b})$, and then obtain a bound on the bit error probability. The PEP is given by

$$
\begin{aligned}
P(\mathbf{b} \rightarrow \overline{\mathbf{b}})=\operatorname{Pr}\left\{\sum_{j=1}^{N}\left\|\mathbf{F} \mathbf{H}^{(j)}(\mathbf{b}-\tilde{\mathbf{b}})+\mathbf{n}^{(j)}-\mathbf{N}_{p}^{(j)} \mathbf{B}_{p}^{T}\left(\mathbf{B}_{p} \mathbf{B}_{p}^{T}\right)^{-1} \tilde{\mathbf{b}}\right\|^{2}\right. \\
\left.-\left\|\mathbf{n}^{(j)}-\mathbf{N}_{p}^{(j)} \mathbf{B}_{p}^{T}\left(\mathbf{B}_{p} \mathbf{B}_{p}^{T}\right)^{-1} \mathbf{b}\right\|^{2}<0\right\},(15)
\end{aligned}
$$

Define the metric $D$ as

$$
D=\sum_{j=1}^{N}\left\|\mathbf{u}^{(j)}\right\|^{2}-\left\|\mathbf{v}^{(j)}\right\|^{2}
$$

where

$$
\begin{aligned}
\mathbf{u}^{(j)} & =\mathbf{F} \mathbf{H}^{(j)}(\mathbf{b}-\tilde{\mathbf{b}})+\mathbf{n}^{(j)}-\mathbf{N}_{p}^{(j)} \mathbf{B}_{p}^{T}\left(\mathbf{B}_{p} \mathbf{B}_{p}^{T}\right)^{-\mathbf{l}} \tilde{\mathbf{b}} \\
& =\mathbf{F} \mathbf{H}^{(j)}(\mathbf{b}-\tilde{\mathbf{b}})+\mathbf{n}^{(j)}-\mathbf{N}_{p}^{(j)} \tilde{\mathbf{c}}, \\
\mathbf{v}^{(j)} & =\mathbf{n}^{(j)}-\mathbf{N}_{p}^{(j)} \mathbf{B}_{p}^{T}\left(\mathbf{B}_{p} \mathbf{B}_{p}^{T}\right)^{-1} \mathbf{b} \\
& =\mathbf{n}^{(j)}-\mathbf{N}_{p}^{(j)} \mathbf{c}, \\
\tilde{\mathbf{c}} & =\mathbf{B}_{p}^{T}\left(\mathbf{B}_{p} \mathbf{B}_{p}^{T}\right)^{-1} \tilde{\mathbf{b}} \\
\mathbf{c} & =\mathbf{B}_{p}^{T}\left(\mathbf{B}_{p} \mathbf{B}_{p}^{T}\right)^{-1} \mathbf{b} .
\end{aligned}
$$


Eqn. (16) can be written in the form

$$
D=\mathbf{V}^{\dagger} \mathbf{S V}
$$

where

$$
\begin{gathered}
\mathbf{V}=\left(\begin{array}{c}
\mathbf{u}^{(\mathbf{1})} \\
\vdots \\
\mathbf{u}^{(N)} \\
\mathbf{v}^{(1)} \\
\vdots \\
\mathbf{v}^{(N)}
\end{array}\right), \\
\mathbf{S}=\left[\begin{array}{cc}
\mathbf{I}_{Q K N} & \mathbf{0} \\
\mathbf{0} & -\mathbf{I}_{Q K N} .
\end{array}\right] .
\end{gathered}
$$

The decision variable $D$ in (18) is in Hermitian quadratic form in the complex Gaussian random vector $V$. This form, from a result in [13], allows us to write the characteristic function of $D, \Phi_{D}(j \omega)$, in closed-form. In order to do that, let

$$
\mathbf{T}=E\left[\mathbf{V} \mathbf{V}^{\dagger}\right] \text {. }
$$

To evaluate $\mathbf{T}$ in the above, we write $\mathbf{H}^{(j)} \mathbf{b}$ in an altemate form [2]

$$
\mathbf{H}^{(j)} \mathbf{b}=\mathbf{B h}^{(j)}
$$

where $\mathbf{B}$ is a $Q K \times Q K$ matrix, which for $M=Q=8$ is defined as

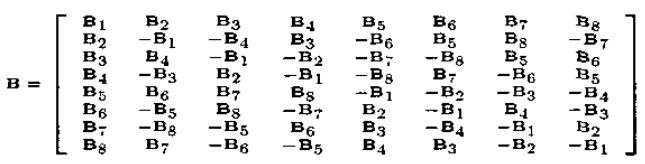

where $\mathbf{B}_{q}=\mathbf{A}_{q} \operatorname{diag}\left\{\mathbf{b}_{q}\right\}, \mathbf{A}_{q}=\operatorname{diag}\left\{A_{1 q}, A_{2 q}, \cdots, A_{K q}\right\}$, $q=1,2, \cdots, Q$. For values of $M$ and $Q$ other than $8,(M, Q<$ 8) B is obtained as follows. For $M=Q \in\{1,2,4\}$, B is given by the upper leftmost submatrix of order $Q K \times Q K$ in (23). For $M \notin\{1,2,4,8\}, M<Q$. In this case, $\mathbf{B}$ is given by the $Q K \times Q K$ upper leftmost submatrix in (23) with all the entries in the $q^{\text {th }}$ column $(M<q \leq Q)$ as zeros. Defining $\mathbf{h}_{q}=$ $\left[h_{1 q}, h_{2 q}, \cdots, h_{K q}\right]^{T}$ and $\mathbf{h}=\left[\mathbf{h}_{1}^{T}, \mathbf{h}_{2}^{T}, \cdots, \mathbf{h}_{Q}^{T}\right]^{T}, E[\mathbf{h}]=$ $\mathbf{0}_{Q K \times 1}$ and $E\left[\mathbf{h h}^{\dagger}\right]=\Omega \mathbf{I}_{Q K}$. Also, let $\beta=\left(1+\tilde{\mathbf{c}}^{T} \tilde{\mathbf{c}}\right), \kappa=$ $\left(1+\tilde{\mathbf{c}}^{T} \mathbf{c}\right)$ and $\epsilon=\left(1+\mathbf{c}^{T} \mathbf{c}\right)$. With the above definitions, we obtain

$$
\begin{aligned}
E\left[\mathbf{u}^{(i)} \mathbf{u}^{(j)^{\dagger}}\right]= \begin{cases}\mathbf{0} \\
\Omega \mathbf{F}(\mathbf{B}-\tilde{\mathbf{B}})(\mathbf{B}-\overline{\mathbf{B}})^{T} \mathbf{F}^{T} & i \neq j \\
+2 \sigma^{2} \beta \mathbf{I}_{Q K} & i=j\end{cases} \\
E\left[\mathbf{u}^{(i)} \mathbf{v}^{(j)^{\dagger}}\right]= \begin{cases}\mathbf{0} & i \neq j \\
2 \sigma^{2} \kappa \mathbf{I}_{Q K} & i=j\end{cases} \\
E\left[\mathbf{v}^{(i)} \mathbf{u}^{(j)^{\dagger}}\right]= \begin{cases}\mathbf{0} & i \neq j \\
2 \sigma^{2} \kappa \mathbf{I}_{Q K} & i=j\end{cases} \\
E\left[\mathbf{v}^{(i)} \mathbf{v}^{(j)^{\dagger}}\right]= \begin{cases}\mathbf{0} & i \neq j \\
2 \sigma^{2} \in \mathbf{I}_{Q K} & i=j,\end{cases}
\end{aligned}
$$

from which $\mathbf{T}$ can be evaluated. Now, the the characteristic function of $D, \Phi_{D}(j \omega)$ can be written as (Ref. [13], Eqn. $(4 . a))$

$$
\Phi_{D}(j \omega)=\frac{1}{\left|\mathbf{I}_{2 N Q K}-2 j \omega \sigma^{2} \mathbf{G}\right|},
$$

where $\mathbf{G}=$ TS. From (20), (21), (24), (25), we can write $\mathbf{G}$ as

0-7803-8255-2/04/\$20.00 @2004 IEEE.
$\mathrm{G}=\left[\begin{array}{cc}\mathbf{I}_{N} \otimes\left(\frac{\Omega}{2 \sigma^{2}} \mathbf{F}(\mathbf{B}-\check{\mathbf{B}})(\mathbf{B}-\check{\mathbf{B}})^{T} \mathbf{F}^{T}+\beta \mathbf{I}_{Q K}\right) & -\mathbf{I}_{N} \otimes \kappa \mathrm{I}_{Q K} \\ \mathbf{I}_{N} \otimes \kappa \mathbf{I}_{Q K} & -\mathbf{1}_{N} \otimes \in \mathbf{I}_{Q K}\end{array}\right]$.

Defining $\widehat{\mathbf{G}}$ as

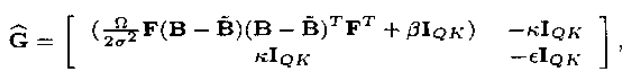

(28) can be written as

$$
\begin{aligned}
\Phi_{D}(j \omega) & =\frac{1}{\left|\mathbf{I}_{2 Q K}-2 j \omega \sigma^{2} \widehat{\mathbf{G}}\right|^{N}} . \\
& =\prod_{i=1}^{2 Q K} \frac{1}{\left|1-2 j \omega \sigma^{2} \widehat{\lambda}_{i}\right|^{N}} .
\end{aligned}
$$

where $\hat{\lambda}_{1}, \cdots, \widehat{\lambda}_{2 Q K}$ are the eigenvalues of $\widehat{\mathbf{G}}$. For the case when the amplitudes of all bits from all the users are the same, i.e., $A_{i q}=A_{j q}=A, i, j=1,2, \cdots, K, q=1,2, \cdots, Q$, and $M=Q$, (31) can be written in the form

$$
\begin{aligned}
\Phi_{D}(j \omega) & =\frac{1}{\left|\mathbf{I}_{2 K}-2 j \omega \sigma^{2} \overline{\mathbf{G}}\right|^{M N}} \\
& =\prod_{i=1}^{2 K} \frac{1}{\left|1-2 j \omega \sigma^{2} \lambda_{i}\right|^{M N}},
\end{aligned}
$$

where $\tilde{\mathbf{G}}$ is given by

$$
\tilde{G}=\left[\begin{array}{cc}
\frac{\Omega A^{2}}{2 \sigma^{2}} \mathbf{P} \boldsymbol{\Lambda} \mathbf{P}^{T}+\beta \mathbf{I}_{K} & -\kappa \mathbf{I}_{K} \\
\kappa \mathbf{I}_{K} & -\epsilon \mathbf{I}_{K}
\end{array}\right],
$$

where $\mathbf{P}$ is the Cholesky decomposition of the $\mathbf{R}$ matrix (i.e., $\left.\mathbf{R}=\mathbf{P}^{T} \mathbf{P}\right), \boldsymbol{\Lambda}$ is given by

$$
\mathbf{\Lambda}=\frac{1}{A^{2}} \sum_{i=1}^{Q}\left(\mathbf{B}_{i}-\tilde{\mathbf{B}}_{i}\right)^{2},
$$

and $\lambda_{1}, \cdots, \lambda_{2 K}$ are the eigenvalues $\tilde{\mathbf{G}}$. Substituting $z=$ $2 j \omega \sigma^{2}$, we have

$$
\Phi_{D}(z)=\prod_{i=1}^{2 K} \frac{1}{\left(1-z \lambda_{i}\right)^{M N}} .
$$

From the above characteristic function of $D$, the PEP in (??) can be obtained as [16], [9]

$$
P(\mathbf{b} \rightarrow \tilde{\mathbf{b}})=-\sum_{k} \frac{1}{\left(p_{k}-1\right) !} \frac{d^{p_{k}-1}}{d z^{p_{k}-1}}\left\{\left(z-\lambda_{k}\right)^{p_{k}} \frac{\Phi_{D}(z)}{z}\right\},
$$

where $\lambda_{k}$ are the negative eigenvalues of $\tilde{G}, \operatorname{Re}\left(\lambda_{k}\right)<0$, and $p_{k}$ is the multiplicity of $\lambda_{k}$. We obtain (36) in closed-form as follows. The characteristic equation of $\tilde{\mathbf{G}}$ is given by

$$
\operatorname{det}\left|\lambda \mathbf{I}_{2 K}-\tilde{\mathbf{G}}\right|=\operatorname{det}\left|\begin{array}{cc}
(\lambda-\beta) \mathbf{I}_{K}-\gamma \mathbf{J} & \kappa \mathbf{I}_{K} \\
-\kappa \mathbf{I}_{K} & (\lambda+\epsilon) \mathbf{I}_{K}
\end{array}\right|=0, \quad \text { (37) }
$$

where $\gamma=\frac{\Omega A^{2}}{2 \sigma^{2}}$ is the average SNR, and $\mathbf{J}=\mathbf{P} \boldsymbol{\Lambda} \mathbf{P}^{T}$. Eqn. (37) can be shown to reduce to the form [14]

$$
\operatorname{det}\left|(\lambda-\beta)(\lambda+\epsilon) \mathbf{I}_{K}-\gamma(\lambda+\epsilon) \mathbf{J}+\kappa^{2} \mathbf{I}_{K}\right|=0 .
$$

If $\mu_{1}, \cdots, \mu_{L}$ are the $L$ distinct eigenvalues of $\mathbf{J}$, each with multiplicity $v_{i}$, i.e. $\sum_{i=1}^{L} v_{i}=2 K$, then (38) reduces to $\prod_{i=1}^{L}\left\{\lambda^{2}-\left(\beta-\epsilon+\gamma \mu_{i}\right) \lambda-\left(\beta \epsilon-\kappa^{2}+\gamma \mu_{i} \epsilon\right)\right\}^{v_{i}}=0$.

From Sylvester's Law of Inertia [15], the eigenvalues of $\mathbf{J}$ are non-negative (i.e., $\mu_{i} \geq 0$ ). Hence, the roots of (39) are all real. Denote the negative roots as $\lambda_{j}$, with multiplicities $g_{j}, j=1,2, \cdots, L_{N}$ and the non-negative roots as $\rho_{i}$, with 
multiplicities $r_{i}, i=1,2, \cdots, L_{P}$, so that $\sum_{j} g_{j}+\sum_{i} r_{i}=$ $2 K^{\circ}$. With this, we can now follow the steps similar to the ones in [9], and obtain the closed-form expression for the PEP as

$$
\begin{aligned}
& P(\mathrm{~b}-\tilde{\tilde{b}})=\sum_{j} \frac{\left(-\lambda_{j}\right)^{M N\left(2 K-g_{j}\right)}}{\prod_{i}^{\left(\rho_{i}-\lambda_{j}\right)^{M N r_{i}}} \prod_{k \neq j}^{\left(\lambda_{k}-\lambda_{j}\right)^{M I N g_{k}}}}
\end{aligned}
$$

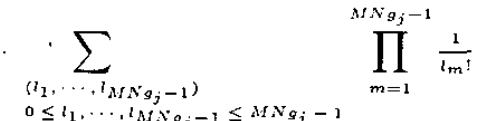

$$
\begin{aligned}
& 0 \leq i_{1}, \ldots, l_{M N g_{g_{j}-1}} \leq M N N_{g_{j}-1} \\
& \begin{array}{l}
l_{1}+2 l_{2}+\cdots+\left(M N_{g_{j}}-1\right) l_{M N g_{j}-1}=M N g_{j}-1 \\
l_{g_{j}}
\end{array} \\
& {\left[\frac{1}{m}+\frac{M N}{m}\left(\sum_{i} \frac{r_{i} \rho_{i}^{m}}{\left(\rho_{i}-\lambda_{j}\right)^{m}}+\sum_{k \neq j} \frac{g_{k} \lambda_{k}^{m}}{\left(\lambda_{k}-\lambda_{j}\right)^{m}}\right)\right]^{l_{m 2}}}
\end{aligned}
$$

where $K$ is the number of users, $M$ is the number of transmit antennas per user, and $N$ is the number of antennas at the receiver.

\section{Bound on the Probability of Bit Error}

Using the expression for PEP in the above, we obtain an upper bound on the bit error probability as follows. Let $\mathbf{b}^{(j)}, 1 \leq$ $j \leq 2^{Q K}$ be the set of $Q K$-bit vectors comprising of $Q$ bits from each of the $K$ users. Suppose $\mathbf{b}^{(k)}$ was the transmitted vector. Define

$$
D_{m}=\sum_{j=1}^{N}\left\|\widehat{\mathbf{y}}^{(j)}-\mathbf{F} \mathbf{H}^{(j)} \mathbf{b}^{(m)}\right\|^{2}, \quad m=1,2, \cdots, 2^{Q K},
$$

where $\widehat{\mathbf{y}}, \mathbf{F}$ and $\mathbf{H}$ are as defined in (13). If $\mathbf{b}^{(l)}$ is the received vector, define

$$
P_{\text {exact }}\left(\mathbf{b}^{(k)} \rightarrow \mathbf{b}^{(l)}\right)=\operatorname{Pr}\left(\bigcap_{\substack{m=1 \\ m \neq l}}^{2^{Q K}}\left(D_{l}<D_{m}\right)\right)
$$

It is noted that the PEP in (40) is nothing but

$$
P\left(\mathbf{b}^{(k)} \rightarrow \mathbf{b}^{(l)}\right)=\operatorname{Pr}\left(D_{l}<D_{k}\right) .
$$

It is clear that

$$
P_{\text {exact }}\left(\mathbf{b}^{(k)} \rightarrow \mathbf{b}^{(l)}\right) \leq P\left(\mathbf{b}^{(k)} \rightarrow \mathbf{b}^{(l)}\right) .
$$

Let $P\left(e_{i q}\right)$ denote the probability of error for the $q^{\text {th }}$ bit of the $i^{t h}$ user, $q=1,2, \cdots, Q$ and $i=1,2, \cdots, K . P\left(e_{i q}\right)$ is then given by

$$
\begin{gathered}
P\left(e_{i q}\right)=\sum_{j=1}^{{ }^{Q K-1}} P\left(e_{i q} \mid \mathbf{b}^{(j)}, b_{i q}^{(j)}=1\right) P\left(\mathbf{b}^{(j)}, b_{i q}^{(j)}=1\right) \\
+\sum_{k=1}^{2 Q K-1} P\left(e_{i q} \mid \mathbf{b}^{(k)}, b_{i q}^{(k)}=-1\right) P\left(\mathbf{b}^{(k)}, b_{i q}^{(k)}=-1\right) .
\end{gathered}
$$

$P\left(e_{i q} \mid \mathbf{b}^{(j)}, b_{i q}^{(j)}= \pm 1\right)$ and $P\left(\mathbf{b}^{(j)}, b_{i q}^{(j)}= \pm 1\right)$ are then given by

$$
P\left(e_{i q} \mid b^{(j)}, b_{i q}^{(j)}=1\right)=\sum_{k=1}^{2 Q K-1} P_{\text {exact }}\left(\mathbf{b}^{(j)}-\mathbf{b}^{(k)} \mid b_{i q}^{(j)}=1, b_{i q}^{(k)}=-1\right), \quad \text { (46) }
$$

$$
\begin{gathered}
P\left(e_{i q} \mid \mathbf{b}^{(k)}, b_{i q}^{(k)}=-1\right)=\sum_{j=1}^{2 Q K-1} P_{\text {exact }}\left(\left.\mathbf{b}^{(k)} \rightarrow \mathbf{b}^{(j)}\right|_{i q} ^{(j)}=1, b_{i q}^{(k)}=-1\right), \\
P\left(\mathbf{b}^{(j)}, b_{i q}^{(j)}=1\right)=P\left(\mathbf{b}^{(k)} \cdot b_{i q}^{(k)}=-1\right)=\frac{1}{2 Q K}
\end{gathered}
$$

From (44) (45),(46),(47) and(48), an upper bound on the bit error probability $P\left(e_{i q}\right)$ is obtained as

$$
\begin{aligned}
P\left(e_{i q}\right) \leq & \frac{1}{2 Q K}\left[\sum_{j=1}^{2 Q K-1} \sum_{k=1}^{2 Q K-1} P\left(b^{(j)}-\left.b^{(k)}\right|_{i q} ^{(j)}=1, b_{i q}^{(k)}=-1\right)\right. \\
& \left.+\sum_{k=1}^{2 Q K-1} \sum_{j=1}^{2 Q K^{K}-1} P\left(\mathbf{b}^{(k)}-\left.b^{(j)}\right|_{i q} ^{(k)}=1, b_{i q}^{(j)}=-1\right)\right]
\end{aligned}
$$

\section{RESULTS AND DISCUSSION}

In this section, we present the numerical results of the error performance of the ML multiuser detection scheme. Fig. 1 shows the PEP plots for the cases of both perfect channel estimation as well as imperfect channel estimation, for a two user system $(K=2)$, with two transmit antennas $(M=2)$ at each user, and one antenna at the receiver $(N=1)$. The correlation coefficient between the two users' signature waveforms, $\rho=0.2$. The power imbalance between the two users is characterized by the near-far ratio (NFR) ${ }^{1}$. In Fig. 1, the NFR is taken to be $0 \mathrm{~dB}$ (i.e., equal power users). It can be seen that, as expected, the PEP degrades with imperfect channel estimation compared to the perfect channel estimates case. These plots are from the PEP exact expressions given in (40). We also verified the correctness of these plots through simulation results, which were found to match with the analytical results. Fig. 2 presents the bit error rate performance obtained through the analytical bound as well as simulations for $K=2$, $M=2, N=1$ and NFR $=0 \mathrm{~dB}$. Plots for both perfect as well as imperfect channel estimates are shown. It can be observed that the analytical bounds become increasingly tight for large SNR values. Also, imperfect channel estimates are seen to degrade the BER performance. For example, at a BER of $10^{-2}$, the performance loss is about $4 \mathrm{~dB}$ in the case of imperfect channel estimation, compared to the perfect channel estimation case. Figs. 3 and 4 shows the bound on the BER as a function of average SNR for $M=2, N=1,2$, and $N=2$, $M=1,2$, respectively, both for the cases of perfect as well as imperfect channel estimates. From Figs. 3 and 4, it is seen that the degradation in BER performance due to imperfect channel estimates can be compensated by using more number of receive/transmit antennas.

\section{Conclusion}

We analyzed the bit error performance of maximum-likelihood (ML) multiuser detection in space-time coded CDMA systems. We considered a $K$-user synchronous CDMA system which employs orthogonal space-time block coding with $M$ transmit antennas and $N$ receive antennas. We derived a closedform exact expression for the pairwise error probability, using

${ }^{1}$ We define NFR as $10 \log \frac{\sum_{p=1}^{M} A_{2 p}^{2}}{\sum_{p=1}^{M} A_{1 p}^{2}}$ assuming $\Omega=E\left|h_{i q}\right|^{2}=1$. 


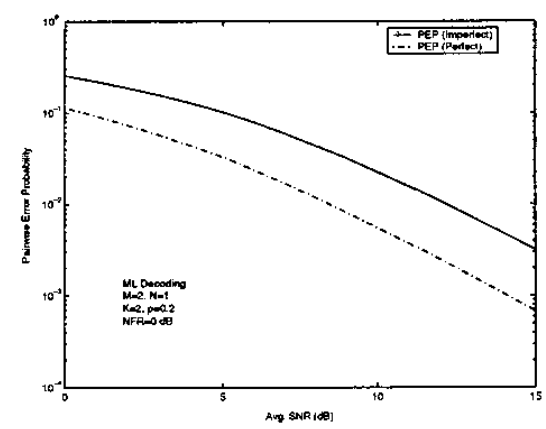

Fig. 1. Pairwise error probability as a function of average SNR for $K=$ $2, M=2 . N=1, \mathrm{NFR}=0 \mathrm{~dB}$. Cases of perfect as well as imperfect channel estimates.

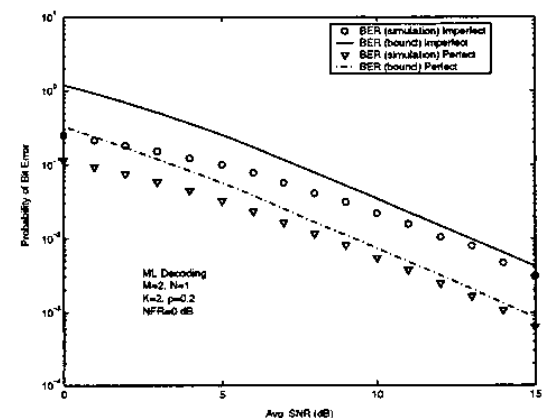

Fig. 2. Bit error rate performance as a function of average SNR for. $K=$ $2, M=2, N=1$. NFR $=0 \mathrm{~dB}$. Analytical bound versus simulations. Cases of perfect as well as imperfect channel estimates.

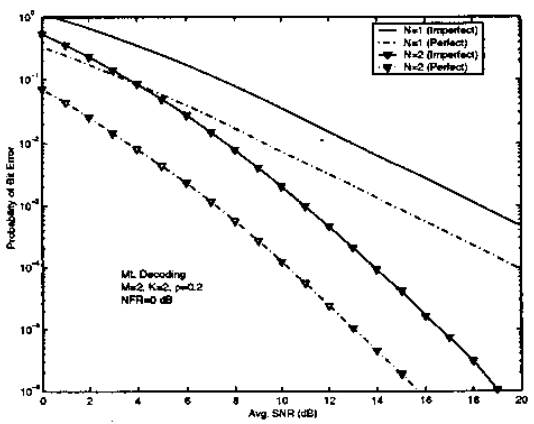

Fig. 3. Bit error probability bound as a function of average SNR for differen number of $\mathrm{Rx}$ antennas, $N=1,2 . K=2, M=2, \mathrm{NFR}=0 \mathrm{~dB}$. Cases of perfect as well as imperfect channel estimates.

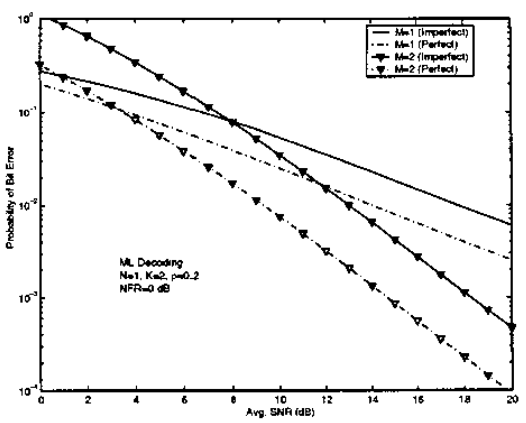

Fig. 4. Bit error probability bound as a function of average $\mathrm{SNR}$ for different number of Tx antennas, $M=1,2 . K=2, N=1, N F R=0 \mathrm{~dB}$. Cases of perfect as well as imperfect channel estimates.

which we obtained an upper bound on the bit error probability. We showed that the analytical BER bounds are increasingly tight for large SNR values.

\section{REFERENCES}

(1) V, Tarokh, N. Seshadri, and A. R. Calderbank. "Space-time codes for high data rate wireless communications: Performance criterion and code construction," IEEE Trans. Inform. Theory, vol. 44, no. 2, pp. 744-765, March 1998.

[2] B. Hochwald, T. L. Marzetta, and C. B. Papadias, "A transmitter diversity scheme for wideband CDMA systems based on space-time spreading," IEEE Jl. Sel. Areas Commun., vol. 19. no. 1, pp. 48-60, January ing," $I$

[3] S. Verdu, Multiuser Detection, Cambridge University Press, 1998

[4] H. Huang, H. Viswanathan, and G. J. Foschini, "Multiple antennas in cellular CDMA systems: Transmission, detection, and spectral efficiency." IEEE Trans. Wireless Commun., vol. 1, no. 3, pp. 383-392, July 2002.

[5] D. Reynolds, X. Wang, and H. V. Poor, "Blind adaptive space-time multiuser detection with multiple transmitter and receiver antennas," IEEE Trans. Sig. Proc., 50(6), pp. 1261-1276, June 2002.

[6] M. M. Alam and A. Chockalingam, "Performance of adaptive multiuser detectors in space-time coded CDMA systems," IEEE PIMRC'2003, September 2003.

[7] M. Uysal and C. N. Georghiades, "Performance of space-time coded CDMA systems for wireless communication," Electronic Letters, vol. 36 , no. 14, pp. 1231-1232, July 2000 .

[8] G. Taricco and E. Biglieri, "Exact pairwise error probability of spacetime codes," IEEE Trans. Inform. Theory, vol. 48, iss. 2, pp. 510-513, Febnary 2002.

[9] P. Garg, R. K. Mallik, and H. M. Gupta, "Performance analysis of spacetime coding with imperfect channel estimation." IEEE ICPWC'2002. pp. $71-75$, December 2002.

[10] G. V. V. Sharma and A. Chockalingam. "Performance analysis of maximum-likelihood multiuser detection in space-time coded CDMA," NCC'2004, January 2004.

[11] M. M. Alam and A. Chockalingam, "Performance of multistage interference cancellers in space-time coded coded CDMA systems," IEEE TENCON'2003, October 2003.

[12] V. Tarokh, H. Jafarkhani, and A. R. Calderbank, "Space-time block codes from orthogonal designs," IEEE Trans. Inform. Theory, vol. 45, no. 5, pp. 1456-1467, July 1999.

[13] G. L. Turin, "The characteristic function of Hermitian quadratic forms in complex normal variables," Biometrika. vol. 47, pp. 199-201, 1960.

[14] D. C. Lay, Linear Algebra and its Applications, Addison Wesley, 2000.

[15] G. Strang, Linear Algebra and its Applications, Harcourt, Brace, Jovanovich, 1988.

[16] J. Gil-Pelacz, "Note on the inversion theorem," Biometrika, vol. 38, pp. 481-482, 1951. 